\title{
Robotic enucleation of a pancreatic uncinate neuroendocrine tumor - a unique parenchyma-saving strategy for uncinate tumors
}

\author{
Ken Min Chin ${ }^{1}$ and Brian K. P. Goh ${ }^{1,2}$ \\ ${ }^{1}$ Department of Hepatopancreatobiliary and Transplant Surgery, Singapore General Hospital, \\ ${ }^{2}$ Duke-National University of Singapore (NUS) Medical School, Singapore
}

\begin{abstract}
Pancreatic neuroendocrine tumors (PNET) comprise up to $10 \%$ of all pancreatic solid tumors. There has been much interest in recent years with regards to the role of limited resection and enucleation procedures for this entity. There is no clear guideline today on the optimal type choice of surgery for this condition, with even fewer reporting on the use of a robotic approach for pancreatic uncinate lesions. We describe a case report of a 54-year-old lady who underwent successful robotic enucleation of pancreatic uncinate neuroendocrine tumor. This patient's recovery was complicated by pancreatitis and a peripancreatic collection, both of which resolved without surgical re-intervention. A literature review was performed with regards to current guidelines on management of PNETs, comparisons between demolitive and parenchymal-preserving procedures, and recent developments in the laparoscopic and robotic approaches for this condition. There is no clear guideline on the optimal type and approach (open vs. laparoscopic vs. robotic) to the surgical management of PNET. We document in this case report a novel approach of robotic enucleation of pancreatic uncinate process NET, that could be considered as an alternative to open/laparoscopic demolitive procedures for small uncinate tumors. (Ann Hepatobiliary Pancreat Surg 2020;24:97-103)
\end{abstract}

Key Words: Pancreas; Uncinate; Neuroendocrine; Robotic; Enucleation

\section{INTRODUCTION}

Pancreatic neuroendocrine tumors (PNET) are the second most common pancreatic neoplasm, comprising up to $10 \%$ of all pancreatic solid tumors. ${ }^{1}$ The diagnosis of PNETs have increased tremendously over the past decade due to the increasingly liberal use of cross-sectional imaging in the workup of non-specific abdominal symptoms. ${ }^{1,2}$ While surgical resection is undoubtedly indicated in functional PNETs (F-PNET), there remains no clear guideline on the indications for surgery in non-functional PNETs (NF-PNET).

Much interest has been directed in recent years towards the role of limited resection and enucleation procedures for proximal low-grade PNETs to avoid the morbidity of major pancreatic resections/pancreaticoduodenectomies. There, however, remains no clear guideline on the optimal type of surgery for this condition (major pancreatic re- sections vs. pancreas-preserving procedures). Literature regarding the surgical approach towards resection of PNET is even more scanty, with a glaring shortage of high powered studies comparing the open, laparoscopic and robotic approaches. Our study is one of few to report the use of the robotic technique for enucleation of a pancreatic uncinate NET.

\section{CASE}

This case involves a 54-year-old lady with no significant past medical history. She presented with symptoms of dyspepsia and gastritis, and was worked up with an ultrasound (US) of the hepatobiliary system. This revealed a $2 \mathrm{~cm}$ isoechoic lesion within the pancreatic uncinate process with non-dilated pancreatic and common bile ducts. No gallstones were seen and there were no other abnormalities of the hepato-pancreato-biliary system.

Received: March 29, 2019; Revised: July 28, 2019; Accepted: July 28, 2019

Corresponding author: Brian K. P. Goh

Department of Hepatopancreatobiliary and Transplant Surgery, Singapore General Hospital, 20 College Road, Academia, 169856, Singapore Tel: +65-6321-4051, Fax: +65-6220-9323, E-mail: bsgkp@hotmail.com

Copyright (C) 2020 by The Korean Association of Hepato-Biliary-Pancreatic Surgery

This is an Open Access article distributed under the terms of the Creative Commons Attribution Non-Commercial License (http://creativecommons.org/ censes/by-nc/4.0) which permits unrestricted non-commercial use, distribution, and reproduction in any medium, provided the original work is properly cited. Annals of Hepato-Biliary-Pancreatic Surgery • pISSN: 2508-5778 - elSSN: 2508-5859 
(Fig. 1A) Further investigation with computer tomography (CT) of the pancreas showed a solid hypervascular mass measuring $1.7 \times 1.5 \times 1.3 \mathrm{~cm}$ in the posteromedial aspect of the pancreatic uncinate process with no invasion into adjacent structures (Fig. 1B). Magnetic resonance cholangiopancreatography (MRCP) confirmed the above findings and measured a distance of $4 \mathrm{~mm}$ between the uncinate process tumor and the adjacent non-dilated main pancreatic duct (MPD) (Fig. 1C). Position emission tomography (PET-CT) showed a fluorodeoxyglucose (FDG)-avid mass in the pancreatic uncinate process suspicious for NET with no distant sites of metastasis (Fig. 1D). An endoscopic retrograde cholangiopancreatography (ERCP) with endoscopic ultrasound (EUS) was performed showing a $1.7 \times$ $1.0 \mathrm{~cm}$ hypoechoeic uncinate process mass with no extension into surrounding structures (Fig. 2A). Endoscopic findings and histopathological analysis from fine needle aspiration cytology (FNAC) were both highly suspicious for NET. Pre-operative tumor markers (carcinoembryonic antigen, cancer antigen 19-9) were within normal ranges, and serum chromogranin A was $63.5 \mathrm{ng} / \mathrm{ml}$.

This patient was counseled for and underwent robotic enucleation of pancreatic uncinate neuroendocrine tumor.

ERCP with endoscopic insertion of an MPD stent was performed prior to surgery. The MPD was cannulated using the sphincterotome with guidewire and deep cannulation was successful with a single pass. Contrast was injected and a pancreatogram obtained. A 5 French $\times 8 \mathrm{~cm}$ Cook Zimmon ${ }^{\circledR}$ single pigtail plastic stent was deployed and unflanged (Fig. 2B). The operative procedure began with insertion of a subumbilical $1.2 \mathrm{~cm}$ port using Hasson's technique. $1.2 \mathrm{~cm}$ right iliac fossa, $0.8 \mathrm{~cm}$ right flank, $0.8 \mathrm{~cm}$ left iliac fossa and $0.8 \mathrm{~cm}$ left flank ports were then inserted under direct vision. Surgery started with mobilization of the hepatic flexure and opening of the gastrocolic ligament with entrance into the lesser sac. Duodenum was kocherized past the aorta and duodenojejunal flexure mobilized. The gastrocolic trunk was divided and vessel loops placed around the middle colic vessels, superior mesenteric artery and vein. Uncinate process of the pancreas was exposed and lesion marked using intra-operative ultrasound (IOUS), confirmed intra-operatively to be 4 $\mathrm{mm}$ away from the MPD and with no extension into gastroduodenal artery, portal vein or bile ducts. Enucleation

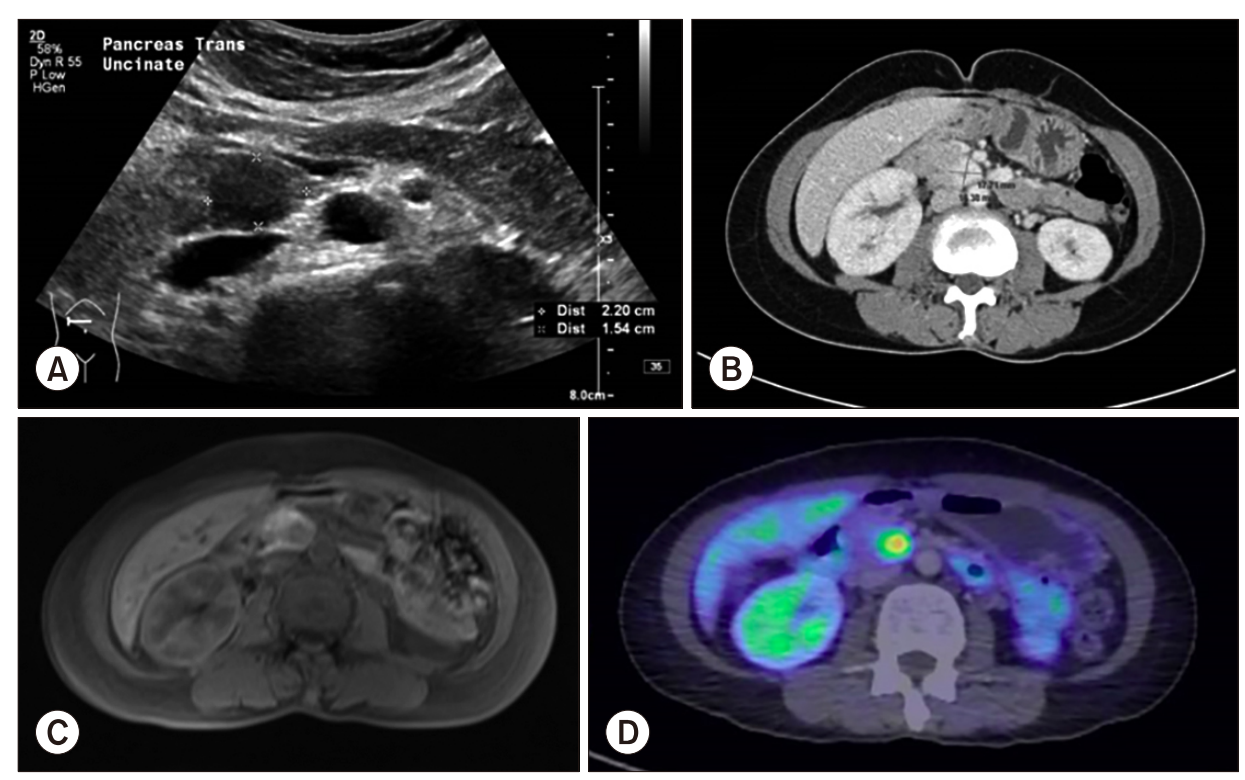

Fig. 1. (A) Ultrasound of the hepatobiliary system showing a $2.2 \times 1.8 \times 1.5 \mathrm{~cm}$ isoechoic lesion within the pancreatic uncinate process with non-dilated pancreatic duct. (B) Computer tomography of the pancreas showing a solid hypervascular mass measuring $1.7 \times 1.5 \times 1.3 \mathrm{~cm}$ in the posteromedial aspect of the pancreatic uncinate process with no invasion into adjacent structures. There is no suspicious lymphadenopathy identified. (C) Magnetic resonance cholangiopancreatography confirming findings of ultrasound hepatobiliary system and computer tomography of the pancreas with a measured distance of $4 \mathrm{~mm}$ between the uncinate process tumor and the adjacent non-dilated main pancreatic duct. (D) Position emission tomography-Computer tomography shoring a fluorodeoxyglucose-avid mass in the pancreatic uncinate process suspicious for neuroendocrine tumor with no distant sites of metastasis. 

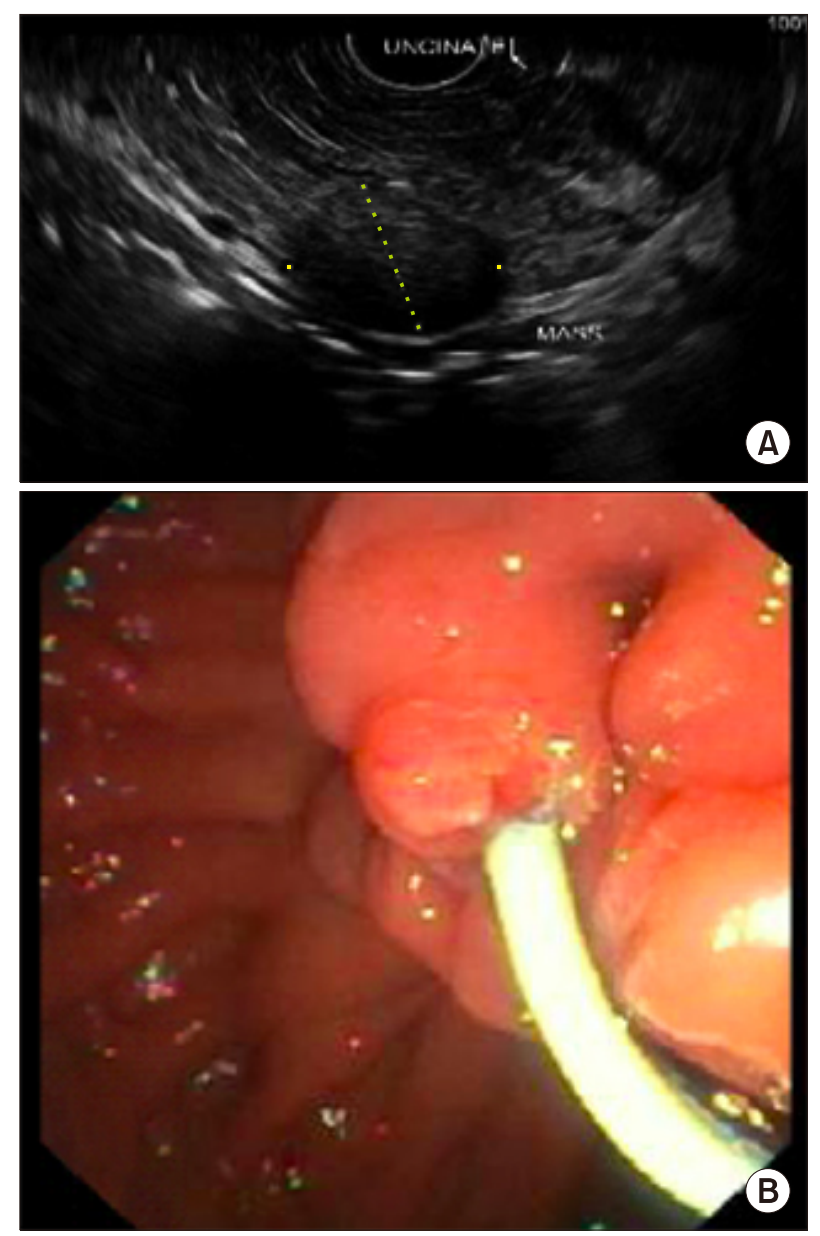

Fig. 2. (A) Endoscopic retrograde cholangiopancreatography with endoscopic ultrasound showing a $1.7 \times 1.0 \mathrm{~cm}$ hypoechoeic uncinate process mass with no extension into surrounding structures. (B) 5 French $\times 8 \mathrm{~cm}$ Cook Zimmon ${ }^{B}$ single pigtail plastic stent was deployed and unflanged within main pancreatic duct.

was completed with Harmonic ${ }^{\circledR}$ scalpel and bipolar electrocautery (Fig. 3). Large tributaries and supplying vessels were divided between clips and hemostasis achieved with Evicel $^{\circledR}$ and Surgicel Snow ${ }^{\circledR}$. Omentum was tagged to the cavity and 2 Blake drains were left in-situ. The total operation time was 325 minutes and estimated blood loss was $50 \mathrm{mls}$.

This patient's post-operative recovery was complicated by raised serum amylase and high drain output with raised fluid amylase. Somatostatin was started and a CT abdomen pelvis (CTAP) performed on post-operative day (POD) 3 revealed a grossly edematous pancreatic body and tail with peripancreatic fluid, compatible with pancreatitis likely secondary to the pancreatic duct stenting. No other abnormalities were detected in adjacent vessels and solid

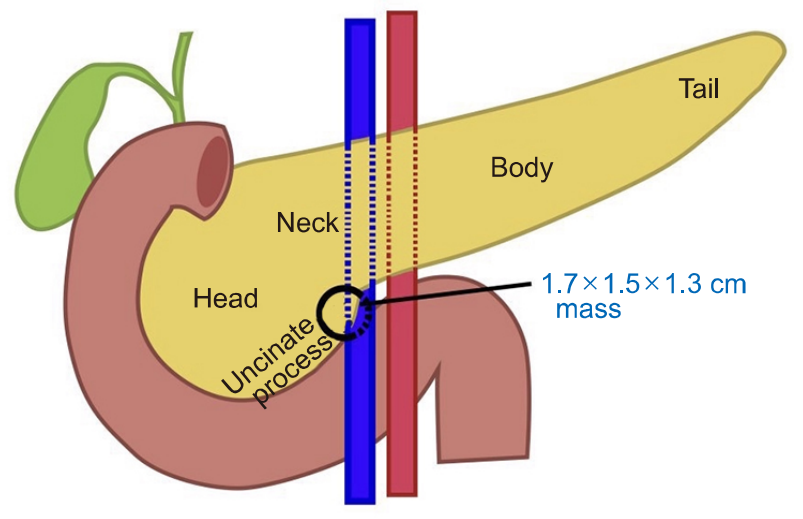

Fig. 3. Relationship of pancreatic uncinate mass to surrounding structure and depicting the extent of resection.

organs. Our patient was managed conservatively with nonopioidal analgesia and aggressive fluid resuscitation. She had resolution of pancreatitis, normalized serum amylase and downtrending drain output by POD 6. A repeat CTAP was performed on POD 10 for persistently high nasogastric tube output with suspicion of mechanical obstruction and gastroparesis. This revealed a $6.5 \times 6.0 \times 4.1 \mathrm{~cm} \mathrm{locu-}$ lated hypodense fluid collection in the surgical bed suggestive of a post-surgical peripancreatic collection. The patient remained hemodynamically stable and was treated with US-guided percutaneous drainage with fluid cultures showing no bacterial growth. She was gradually escalated to feeds on POD 12 and subsequently tolerated diet on POD 14. Nasogastric tube and surgical drains were removed on POD 15 and 16 respectively and a repeat CTAP on POD 24 showed complete resolution of peripancreatic collection. She was discharged stable, afebrile and asymptomatic on POD 18.

Histology revealed a well-differentiated, grade 1 uncinate neuroendocrine tumor, $1.6 \mathrm{~cm}$ in size. Mitotic rate was $1 / 2 \mathrm{~mm}^{2}$ and Ki-67 labeling index was $<3 \%$. There was no peripancreatic extension of tumor, tumor necrosis, lymphovascular or perineural invasion. Immunohistochemistry revealed positivity for synaptophysin but negative for chromogranin. On last follow-up 16 months postoperatively, there was no evidence of recurrence and a complete return to functional baseline.

\section{DISCUSSION}

PNETs are rare neoplasms comprising $5-10 \%$ of all di- 
agnosed pancreatic tumors, with an estimated prevalence of 1 in 100,000 people. ${ }^{1}$ The diagnosis of this condition has increased six fold over the past decade, largely owing to incidental diagnoses from more frequent use of high resolution radiological imaging for work-up of non-specific abdominal symptoms. ${ }^{1}$ Furthermore, the size of diagnosed PNETs has considerably decreased, with a sizeable number being detected at $<2 \mathrm{~cm}(26-61 \%){ }^{2}$ PNETs can be divided into NF-PNET and F-PNET tumors (insulinoma, gastrinoma, glucagonoma, VIPoma, somatostatinoma), with the majority belonging to the former group (90\%). ${ }^{1}$

Mitotic count and Ki-67 expression are widely recognized as the most important factors differentiating between the grades of PNETs, with significant implications on prognosis. While there has been no universally agreed Ki-67 cutoff differentiating the grades of PNETs, the 2017 World Health Organization (WHO) classification states $3 \%$ and $20 \%$ as being appropriate cutoffs between Grade 1-3 PNETs. ${ }^{1,3,4}$ Numerous prognostication systems have been proposed for PNETs, with the ones most commonly used today being the WHO 2010 criteria, European Neuroendocrine Tumor (ENET) society guidelines and the American National Comprehensive Cancer Network (NCCN) guidelines. Across most guidelines, the most commonly cited prognostic factors include age, size, lymphatic metastasis, peritumoral invasion, distant metastases, calcification on imaging, absence of symptoms in NF-PNETs, Ki-67 index and mitotic count. ${ }^{5,6}$ There is no gold standard for management of NF-PNETs, with marked differences between a multitude of different guidelines. The European Neuroendocrine Tumor (ENET) Society guidelines states that patients with G1/G2 NF-PNETs $<2 \mathrm{~cm}$ could be managed conservatively in the presence of pancreatic head localization and no signs of malignancy at initial imaging. These guidelines also mandate biannual EUS and MRCP with re-evaluation for surgery once there is an increase in tumor size of $0.5 \mathrm{~cm}^{3}$ In contrast, the American National Comprehensive Cancer Network (NCCN) guidelines suggest conservative management only for incidentally diagnosed, low grade NF-PNETs that are $<1 \mathrm{~cm}^{7}$ The Canadian National Expert Group advocates a surgical approach for every healthy patient with resectable disease, with surveillance only considered in NF-PNETs smaller than $2 \mathrm{~cm}$ with a low Ki-67 index. ${ }^{8}$ Arguments for an aggressive approach towards NF-PNETs include statistics suggesting frankly malignant behaviour in a significant (40\%) portion of tumors smaller than $2 \mathrm{~cm}$, with thorough histological examination (mitotic and Ki-67 indices) possible only on a resected specimen. ${ }^{1}$ This has, however, to be balanced against the considerable morbidity and mortality associated with pancreatic surgery, and long term risks of exocrine and endocrine insufficiency.

The surgical management of PNET is a delicate balance between demolitive (total pancreatectomy, distal pancreatectomy, pancreaticoduodenectomy), and pancreaspreserving surgery (enucleation (EN)). There is no gold standard of guidelines currently that optimizes the morbidity of excessive pancreatic parenchymal removal against benefits of long term disease-free survival (DFS) and overall survival (OS). While there have been sporadic reports of suboptimal results after EN in terms of DFS and OS as compared to demolitive procedures, the vast majority of reviews report no significant differences. ${ }^{1,9-11}$ Most recent NCCN guidelines report benign tumors, isolated lesions, distance between tumor and MPD $>3 \mathrm{~mm}$ with no focal stricture or dilatation, insulinomas and gastrinomas $<2 \mathrm{~cm}$ in size, and NF-PNETs $<2 \mathrm{~cm}$ in size with low $\mathrm{Ki}-67$ and mitotic indices as indications for $\mathrm{EN}$ procedure. ${ }^{7}$ For the group of F-PNETs, EN is advocated only for insulinomas, given the higher risk of lymph node metastasis and locoregional involvement associated with other F-PNET subtypes. ${ }^{6}$ Strict adherence to the size criteria for NF-PNETs is strongly advocated in this study, given the undoubted correlation between tumor size and risk of malignancy and metastasis. ${ }^{7}$ A common consensus of current data suggests that an EN approach is feasible only for small $(<2 \mathrm{~cm})$, low grade, superficial NFPNETs. ${ }^{1,8,10}$ With appropriate selection criteria applied, EN has been shown to have superior perioperative outcomes (operative times, blood loss, adverse events according to Clavien-Dindo grading), and an equivalent DFS and OS when compared to demolitive procedures. ${ }^{1,9-12}$ One major drawback of EN is its consistently significant correlation with higher rates of post-operative pancreatic fistulas (POPF), especially in head of pancreas (HOP) and uncinate PNETs. ${ }^{1}$ Postulated explanations include difficulty with suture re-approximation of pancreatic parenchyma after EN, and PNETs being more commonly associated with a non-dilated MPD within a soft and friable pancreas (specifically higher risk in HOP lesions due to the pres- 
ence of a larger pancreatic duct). ${ }^{1}$ Most current guidelines mandate that tumors should be at least $2-3 \mathrm{~mm}$ from the MPD to reduce risk of direct injuries and POPF development. $^{1,11}$ This should be evaluated pre-operatively with MRCP, and intra-operatively with IOUS. Fortunately, a large proportion of POPF that do occur are classified as low grade and are most commonly amenable to conservative management. ${ }^{1,12,13}$ Enucleation also presents a unique challenge in terms of pancreatic uncinate tumors due to its location and intimate association with important structures (duodenum, pancreaticoduodenal vessels, superior mesenteric vessels) necessitating careful, patient and precise peri-tumoral dissection.

In comparing open and minimally invasive (MIS) approaches to PNET surgery, the latter has been reported to be non-inferior, and significantly associated with shorter operative time, lower morbidity and POPF rates, and decreased duration of hospital stay. ${ }^{12-14}$ To date there exist only two meta-analyses specific to the comparison between MIS and open approaches for PNETs. Both studies have found MIS to be associated with decreased perioperative complications, less blood loss, shorter length of stay with comparative operative time, POPF incidence and operative mortality rates. ${ }^{12}$ In addition, MIS EN was associated with better post-operative outcomes when compared to other MIS parenchymal-preserving procedures (central pancreatectomy, HOP resection, distal pancreatectomy). ${ }^{1,11}$ The vast majority of other well powered studies fail to discriminate non-PNET pathologies, making data poorly translatable to this specific subgroup of patients.

Robotic pancreatic surgery (RPS) has been gaining traction in recent years for the management of both benign and malignant pancreatic conditions. Goh et al. ${ }^{15}$ report on the safe adoption of RPS for a variety of hepatopancreatobiliary surgeries (including distal pancreatectomy, subtotal pancreatectomy, pancreato-splenectomy, Puestow procedure, uncinectomy and pancreaticoduodenectomy) with low rates of open conversion (3.3\%), POPF (13.3\%) and 0 30-day/in-hospital mortalities. While robotic surgery is generally associated with a longer operative time (robot docking/undocking, instrument changing, bias towards a larger proportion of surgeons better versed with the open/laparoscopic approaches as compared to the robotic approach) and increased costs, many argue that this is compensated by lower rates of open conversion, higher rates of splenic preservation, reduced length of hospital stay and quicker return to activities. ${ }^{12,16-19}$ Most studies now report comparable results between robotic and laparoscopic surgery with regards to both peri-operative, long-term and oncological outcomes. ${ }^{12,17-19}$ It is however widely recognized that there is an overall lack of statistically powered studies comparing the open, laparoscopic and robotic techniques for management of PNETs, especially with regards to long term DFS and OS. ${ }^{1,16}$ Moreover, most current studies have marked heterogeneity with regards to tumor type and surgical procedure (pure robotic vs. pure laparoscopic vs. combination procedures), limiting the feasibility of cogent comparison.

Ore et al. ${ }^{17}$ is one of few to have documented a suggested technique for robotic enucleation of PNETs. This technique differed from ours firstly in terms of port placement $(5 \mathrm{~mm}$ port in the right anterior axillary line to secure the liver retractor, $12 \mathrm{~mm}$ port in the right lower quadrant for ultrasound access and needle passage, $12 \mathrm{~mm}$ camera port located in proximity to the tumor and three $8 \mathrm{~mm}$ robotic ports across the upper abdomen). This report also advocated the use of secretin administration with observation of surgical cavity for signs of pancreatic leak. Apart from this, surgical technique and steps were largely similar between this report and ours. ${ }^{17}$ In addition, Ore et al. ${ }^{17}$ performed a systematic review revealing shorter operative time, reduced blood loss and no significant differences in morbidity, post-operative stay or POPF rates when robotic EN was compared to open and laparoscopic techniques. Distance between tumor and MPD as well as operative time were reported to be the only significant predictors for POPF in patients undergoing minimally-invasive EN of PNETs. ${ }^{17}$

A separate propensity score-matched analysis conducted by Tian et al. ${ }^{18}$ found that robotic EN of PNETs for tumors $<2 \mathrm{~cm}$ did not increase risk for POPF or major post-operative complications as compared to the open approach. In addition, this study found that robotic surgery reduced the duration of surgery and blood loss. ${ }^{18}$ Zhang et al. ${ }^{19}$ is one of few to have reported on comparisons between robotic and laparoscopic pancreatectomies for PNETs. This study found the robotic approach to be superior with regards to less blood loss, higher rates of splenic preservation and more lymph node harvest. Gene- 
ralizability is however limited, given that this study only included patients undergoing distal pancreatectomies. ${ }^{19}$ Machado et al. ${ }^{20}$ reported in November 2018 the first case of robotic resection of pancreatic uncinate process for PNET. The distance between tumor and MPD was $4 \mathrm{~mm}$ and this patient had no post-operative complications. ${ }^{20}$

To our knowledge, this is the second report of a robotic enucleation of a pancreatic uncinate NET in the current literature. Although the surgical procedure proceeded smoothly with no major intraoperative adverse events and minimal blood loss, the patient's postoperative recovery was complicated by the development of POPF with a symptomatic intra-abdominal collection requiring percutaneous drainage. This in our opinion was not surprising as $\mathrm{EN}$ is well-known to be associated with a high rate of POPF of over $50 \%$ especially when the tumor is deepseated and located close to the MPD. ${ }^{11,12,14}$ Nonetheless, it is important to note that despite the potentially higher postoperative morbidity rate especially with regard to the POPF associated with EN compared to pancreaticoduodenectomy, the incidence of life-threatening POPF such as bleeding from pseudoaneurysm is frequently lower with EN. This has been postulated to be due to the absence of activated pancreatic juice after EN compared to that after pancreaticoduodenectomy whereby the pancreatic fluid has been mixed with and potentially activated by enteric contents. Moreover, pancreaticoduodenectomy when performed for PNET is well-known to be associated with a higher rate of clinically-significant POPF compared to that for ductal adenocarcinoma due to the presence of a small non-dilated pancreatic duct and soft pancreas. ${ }^{12}$

Hence, when considering performing $\mathrm{EN}$ for uncinate tumors, one should carefully consider the technical challenges of this rare and novel procedure especially via the minimally-invasive approach, combined with the increased incidence of POPF versus the more severe morbidity, mortality and long-term consequences associated with pancreaticoduodenectomy. More comparative studies in larger patient cohorts are needed to determine if $\mathrm{EN}$ is a safe and effective alternative parenchymal-saving surgical strategy for small benign and premalignant tumors located in the uncinate process.

\section{CONCLUSION}

We report a novel case of successful robotic enucleation of pancreatic uncinate process NET. This surgical strategy may be considered as an alternative pancreatic parenchymal-saving approach to pancreatoduodenectomy for small benign tumors in the pancreatic uncinate process. However, further studies are needed to confirm the feasibility and safety of this rare technically-demanding procedure especially when performed via the minimallyinvasive approach.

\section{ACKNOWLEDGEMENTS}

All work was performed at Singapore General Hospital, 1 Hospital Drive, Singapore 169608.

No financial grants or funding were used for this report. This manuscript has not been published in or submitted for publication in any other journal. Subject to acceptance, authors will transfer copyright to the publisher. There is no ethical problem or conflict of interest to declare.

Written informed consent was obtained from the patient for publication. A copy of the written consent is available for review by the Editor-in-Chief of this journal.

\section{ORCID}

Ken Min Chin: https://orcid.org/0000-0002-7954-5489

Brian K. P. Goh: https://orcid.org/0000-0001-8218-4576

\section{AUTHOR CONTRIBUTIONS}

Ken Min Chin: acquisition of data, interpretation of data, drafting manuscript, revision of manuscript. Brian $\mathrm{K}$. P. Goh: acquisition of data, interpretation of data, drafting manuscript, revision of manuscript, final approval of manuscript.

\section{REFERENCES}

1. Bartolini I, Bencini L, Risaliti M, Ringressi MN, Moraldi L, Taddei A. Current management of pancreatic neuroendocrine tumors: from demolitive surgery to observation. Gastroenterol Res Pract 2018;2018:9647247.

2. Cheema A, Weber J, Strosberg JR. Incidental detection of pancreatic neuroendocrine tumors: an analysis of incidence and 
outcomes. Ann Surg Oncol 2012;19:2932-2936.

3. Falconi M, Eriksson B, Kaltsas G, Bartsch DK, Capdevila J, Caplin M, et al.; Vienna Consensus Conference participants. ENETS consensus guidelines update for the management of patients with functional pancreatic neuroendocrine tumors and non-functional pancreatic neuroendocrine tumors. Neuroendocrinology 2016;103:153-171.

4. Lloyd RV, Osamura RY, Klöppel G, Rosai J, Bosman FT, Jaffe ES, et al. WHO classification of tumours of endocrine organs. 4th ed. Lyon: International Agency for Research on Cancer, 2017.

5. Bilimoria KY, Talamonti MS, Tomlinson JS, Stewart AK, Winchester DP, Ko CY, et al. Prognostic score predicting survival after resection of pancreatic neuroendocrine tumors: analysis of 3851 patients. Ann Surg 2008;247:490-500.

6. Teo RYA, Teo TZ, Tai DWM, Tan DM, Ong S, Goh BKP. Systematic review of current prognostication systems for pancreatic neuroendocrine neoplasms. Surgery 2019;165:672-685.

7. Shah MH, Goldner WS, Halfdanarson TR, Bergsland E, Berlin JD, Halperin D, et al. NCCN guidelines insights: neuroendocrine and adrenal tumors, version 2.2018. J Natl Compr Canc Netw 2018;16:693-702.

8. Singh S, Dey C, Kennecke H, Kocha W, Maroun J, Metrakos $\mathrm{P}$, et al. Consensus recommendations for the diagnosis and management of pancreatic neuroendocrine tumors: guidelines from a Canadian National Expert Group. Ann Surg Oncol 2015;22: 2685-2699.

9. Jilesen AP, van Eijck CH, Busch OR, van Gulik TM, Gouma DJ, van Dijkum EJ. Postoperative outcomes of enucleation and standard resections in patients with a pancreatic neuroendocrine tumor. World J Surg 2016;40:715-728.

10. Falconi M, Zerbi A, Crippa S, Balzano G, Boninsegna L, Capitanio $\mathrm{V}$, et al. Parenchyma-preserving resections for small nonfunctioning pancreatic endocrine tumors. Ann Surg Oncol 2010;17:
1621-1627.

11. Zhou Y, Zhao M, Wu L, Ye F, Si X. Short- and long-term outcomes after enucleation of pancreatic tumors: an evidence-based assessment. Pancreatology 2016;16:1092-1098.

12. Teo RYA, Goh BKP. Surgical resection of pancreatic neuroendocrine neoplasm by minimally invasive surgery-the robotic approach? Gland Surg 2018;7:1-11.

13. Beger HG, Siech M, Poch B, Mayer B, Schoenberg MH. Limited surgery for benign tumours of the pancreas: a systematic review. World J Surg 2015;39:1557-1566.

14. Kabir T, Tan ZZX, Syn N, Chung AYF, Ooi LLPJ, Goh BKP. Minimally-invasive versus open enucleation for pancreatic tumours: a propensity-score adjusted analysis. Ann Hepatobiliary Pancreat Surg 2019;23:258-264.

15. Goh BKP, Low TY, Kam JH, Lee SY, Chan CY. Initial experience with laparoscopic and robotic surgery for the treatment of periampullary tumours: single institution experience with the first 30 consecutive cases. ANZ J Surg 2019;89:E137-E141.

16. Bencini L, Annecchiarico M, Farsi M, Bartolini I, Mirasolo V, Guerra F, et al. Minimally invasive surgical approach to pancreatic malignancies. World J Gastrointest Oncol 2015;7:411-421.

17. Ore AS, Barrows CE, Solis-Velasco M, Shaker J, Moser AJ. Robotic enucleation of benign pancreatic tumors. J Vis Surg 2017; 3:151.

18. Tian F, Hong XF, Wu WM, Han XL, Wang MY, Cong L, et al. Propensity score-matched analysis of robotic versus open surgical enucleation for small pancreatic neuroendocrine tumours. $\mathrm{Br}$ J Surg 2016;103:1358-1364.

19. Zhang J, Jin J, Chen S, Gu J, Zhu Y, Qin K, et al. Minimally invasive distal pancreatectomy for PNETs: laparoscopic or robotic approach? Oncotarget 2017;8:33872-33883.

20. Machado MAC, Surjan R, Basseres T, Makdissi F. Robotic resection of the uncinate process of the pancreas. J Robot Surg 2019;13:699-702. 